\title{
Características del castigo físico infantil administrado por padres de tres colegios de Santiago
}

\author{
Nelson A. Vargas C. ${ }^{\text {; }}$ Daniel López S. ${ }^{2}$ Paulina Pérez R. ${ }^{3 .}$ \\ Pamela Zúníga C. ${ }^{3}$; Gloria Toro V. ${ }^{3}$; Paola Ciocca B. ${ }^{4}$

\begin{abstract}
Features of physical maltreatment given by parents to school children in metropolitan Santiago, Chile
\end{abstract}

\begin{abstract}
An ononymous selfodministered closed-questions survey wos applied to 527 parents or tutors of schood children in metropoliton Sontiogo. Chile, lo learn about their opinions ond betwavors on child physical punishment: 173 (32.8\%) parents came from a counly school, 250 (45.5\%) from a government subsidized school (both were free of charge ond nonconfessionali and $104\{19.7 \%$ from a privale, user payed, calholic institulion. Belter educalional level and o higher propollion of surveyed males were recorded among porenls from the lotet. Child bollering was recognized by $53.7 \%$ (private school), $78.0 \%$ (county) and $82 \%$ (subsidized) school parents even though 34 . 1\% [countyl to $51.9 \%$ (private) of them dectated thot bottering should never be used. females odmitted child baltering more frequently than males at hwo shools (p<0.011. Poot school performance, defiant allitudes and running oway from home were the maln reasons alluded to justify battering at both public schools, while defiance was the most frequent cloim from privale school parents. Femoles tended to select o higher number of reasons, from those offered by the questionary, to justify physicol punishment. No striking differences were delected in type of physical punlshment, but among privole school group belt ballering was not admilled.
\end{abstract}

[Key words: child battering, child abuse, violence, lomily heoth.)

En 1993 el Parlamento chileno analizó diversas formas de violencia familiar; entre ellas el maltrato infantil, agresión ejercida sobre un individuo sin gran capacidad de defensa y que no puede comprender, cabalmente, la razón de la violencia. Una forma de maltrato infantil es el castigo físico, cuya prevención debe basarse en el conocimiento de su epidemiología descriptiva y factores condicionantes. Con este raciocinio, el propósito de este estudio fue comparar opiniones

1. Departamento de Salud Pública Occiderte. Departamento de Pediatría y Cingía Infantil Occidente. Facultad de Medicina, Universidad de Chile.

2. Intemo carrera de Medicina. Ayndante alumno. Departamento de Salud Pública Occidente, Facultad de Medj. cina, Universidad de Chite.

3. Programa de Formación de Especialistas en Pedistría, Depanamento de Pediatrí y Cinggia Infanil Occidente, Facultad de Medicina, Universidad de Chile.

3. Intema carrera de Medicina. Facultad de Medicina, Universidad de Chile. y conductas sobre el castigo físico (CF), en padres y apoderados (PyA) de tres colegios de Santiago metropolitano. Los objetivos fueron establecer la proporción que usa el CF, por colegio y por sexo; precisar las razones que, en opinión de los padres, lo justificarían y conocer los castigos más frecuentes. Este artículo completa la información aparecida en otra publicación ${ }^{1}$, donde se detalla parte del método.

\section{Personas, material y Método}

Se usó una encuesta anónima de respuestas cerradas, auyo encabezado excluyó las palabras "castigo" y "malırato", que fue autoeplicada . PyA de cuarto a octavo años básicos, en reuniones regulares y sin explicación oral. para evitar la deliberación. La información sobre CF se obuvo con una pregunta indirecta (" 2 Cómo le pega a sus hijos?"). incluids en una serie de fomas posibles de castigo. Al analizar los datos, se considera que responder esa pregunia lleva implícito el uso de CF. La encuesta se aplicó en 1991, 
en un colegio municipal ( $\mathrm{n}=173 ; 32,8 \%$ ); otro subvencionado $(n=250 ; 45,5 \%)$, ambos de la comuna de Renca, y uno particular $(n=104 ; 19,7 \%)$ de Las Condes. Los primeros son laicos y gratuitos, aunque en el subvencionsdo se paga el centro de padres. El tercero es confesional catolico y se pagan matrícula y mensualidades.

Las difierencias entre porcentajes se estudiaron mediante el cálculo de $Z$, con un rivel de significación de $1 \%$, unileteral. La asociacion entre haber sido golpendo en la infancia, haber sido golpeado por el cónyuge y contestar la pregunta sobre la forma en que se golpea a los niños se estudió con el calculo de las razones de disparidad ("odds ratio").

\section{Resultados}

Respondieron 527 adultos. La edad se distribuyó en forma normal, sin variar por sexo, el promedio osciló entre 38,5 años (hombres) y 35,3 años (mujeres), Al comparar los colegios no hubo diferencias significativas en el estado civil; pero sí en el rivel educacional: la suma de sujetos con educación media completa y universitaria alcanzó a $70,2 \%$ en el colegio particular $(p<0,01), 4,8 \%$, en el subvencionado y $5,8 \%$, en el municipal. La tabla 1 muestra la distribución por sexo de los encuestados. En todos los colegios predominaron las mujeres, en especial en el subvencionado ( $p$ $<0,01)$. La proporción de hombres fue mayor en el particular $(p<0,01)$; mientras la faita de identificación del sexo fue más frecuente en el municipal.

La tabla 2 muestra la aceptación del uso de CF y la proporción de padres y apoderados que respondió la pregunta sobre cómo golpeaba a los niños. La omisión de respuesta a la aceptación del castigo físico fue variable: mayor en el municipal $(23,7 \%)$ y mínima en el paricular $(2,9 \%)$. Este último tuvo la proporción más alta de PyA que estimaron que el CF no debía usarse y la menor proporción de respuestas "a veces" y "a menudo". En todos los colegios hubo falta de coherencia entre la proporción de padres que contestó "nunca es necesario golpear" y la que admitió golpear los niños.

La tabla 3 muestra el porcentaje de PyA que contestó la pregunta sobre la forma en que golpeabi a sus tijos. Este grupo, en el total, llegó a $75,5 \%$. La respuesta fue más frecuente en las mujeres, tanto en el total $(p<0,002)$ como en dos colegios; y sólo en el municipal los hombres mostraron mayor frecucncia de la respuesta. En el colegio particular se obtuvieron las frecuencias más bajas, tanto en hombres como en mujeres.
Tabla 1

Sexo de los padres por colegio. Distribución porcentual

\begin{tabular}{lrrr}
\hline Sexo & Municipal & Particular & $\begin{array}{r}\text { Subvencio- } \\
\text { nado }\end{array}$ \\
\hline Femenino & 71,1 & 64,4 & 80,4 \\
Masculino & 7,5 & 29,8 & 11,6 \\
Ignorado & 21,4 & 5,8 & 8,0 \\
\hline Tocal & 100,0 & 100,0 & 100,0 \\
\hline
\end{tabular}

Tabla 2

Necesidad de uso del castigo físico por colegio y proporción que contesta cómo golpea lcs ninos. Distribución porcentual

\begin{tabular}{lrrr} 
Necesidad & Munlcipal & Particular & $\begin{array}{c}\text { Subvenclo- } \\
\text { nado }\end{array}$ \\
\hline Nunca & 34,1 & 51,9 & 38,4 \\
Rara vez & 20,2 & 33,7 & 20,0 \\
A veces & 21,4 & 11,5 & 26,4 \\
A menudo & 0,6 & 0,0 & 0,8 \\
No responde & 23,7 & 2,9 & 14,4 \\
Proporción que & 78,0 & 56,7 & 82,0 \\
golpeo los nitios & & & \\
\hline
\end{tabular}

Tabla 3

Proporción de padres $y$ apoderados que contestan la pregunta sobre cómo golpea a sus hijos, por colegio y sexo

\begin{tabular}{lcccc}
\hline Sexo & Total & $\begin{array}{c}\text { Muni- } \\
\text { cipal }\end{array}$ & $\begin{array}{c}\text { Part- } \\
\text { cular }\end{array}$ & $\begin{array}{c}\text { Subvenclo- } \\
\text { nado }\end{array}$ \\
\hline Masculino & 54,8 & 84,6 & 38,7 & 58,6 \\
Femenino & 80,8 & 82, & 164,2 & 85,5 \\
Ambos & 75,5 & 78,0 & 56,7 & 82,0 \\
\hline
\end{tabular}

La frecuencia más alta de respuesta se registró en las mujeres del colegio subvencionado y la más baja en los hombres del particular.

Las siluaciones que justificarían el CF (tabla 4) muestran que el particular presenta porcentajes más bajos en todas las razones ofrecidas. En dos colegios, no estudiar, desafiar y escapar de casa ocupan las frecuencias más altas. En el particular la justificación mayor corresponde a la acción desafiante. 
Tabla 4

Situaciones que justificarian el CF: elección entre situaciones ofrecidas. según colegio. Distribución porcentual

\begin{tabular}{lrrrr}
\hline $\begin{array}{l}\text { Situaclón ofrecidan } \\
\text { para justificar }\end{array}$ & Total & $\begin{array}{c}\text { Muabi- } \\
\text { cipal }\end{array}$ & $\begin{array}{l}\text { Parti- } \\
\text { cular }\end{array}$ & $\begin{array}{l}\text { Subven- } \\
\text { clonado }\end{array}$ \\
\hline No estudia & 34,3 & 45,1 & 3,9 & 39,6 \\
Me desafia & 30,4 & 30,6 & 17,3 & 35,6 \\
Escapa de casa & 22,0 & 26,6 & 31,5 & 23,2 \\
No come & 8,0 & 9,8 & 1,0 & 9,6 \\
Rel. scxuales & 3,4 & 4,1 & 4,4 & \\
Otra & $1,4,4$ & 0,6 & 2,8 & \\
\hline
\end{tabular}

El tipo de castigo aplicado se presenta en la tabla 5 . El CF oscila entre $56 \%$ y $82 \%$. El particular tiene una frecuencia menor de $\mathrm{CF}$ ( $\mathrm{p}<$ $0.01)$ y los padres no declaran golpear con correa.

La relación entre haber sido golpeado y golpear fue 77,5 vs $78,0 \%$ en los padres del colegio municipal, 59,6 vs $56,7 \%$ en el privado y 80 vs $82 \%$ en el subvencionado, observándose en collsecuencia parale lismo entre ambas situaciones y cifras más bajas en el colegio particular. El cálculo de las razones de disparidad demostró una asociación significativa $(p<0,01)$, en todos los colegios. La asociación entre haber sido golpeada(o) por el cónyuge y castigar físicamente a los niños no fue significativa en las mujeres ni en el total de padres. La proporción de mujeres que admitió haber sido golpeada por el cónyuge varió significalivamente: $21,1 \%$, en el colegio municipal, $1,5 \%$ en el particular y $11,0 \%$, en el subvencionado.

\section{Comentario}

El análisis de los datos de castigo físico incluye la presunción de homologar una respuesta (contestar cómo se golpea a los niftos) con el uso efectivo del mismo. Ella parece razonable y, más aún, es probable que minimice la frecuencia real de castigo físico, si consideramos que en los ülímos años este ha sido fuertemente criticado en los medios de comunicación de masas. Los hallazgos del estudio reafirman el planteamiento de que la violencia hacia el niño se aprende durante la infancia (la asociación entre haber sido golpeado y golpear fue significativa en todos los colegio) y es modulada por condicionantes de la vida adulta ${ }^{2-4} \mathrm{e}$ insinúan que el CF tiene patrones distintos en los diferentes medios del país. Esta úlima idea se apoya en que el colegio particular representa un estrato socioeconómico diferente, con alta probabilidad de mayor ingreso y, claramente, con mayor educación formal.

La frecuencia de castigo físico fue muy alta en los colegios estudiados $y$, en conjunto, se asemeja a la encontrada en Barbados ${ }^{5}$, país con cultura muy diferente a Chile. Sin embargo, en el análisis por colegios, el establecimiento particular mostró diferencias marcadas con los otros: menor frecuencia de respuesta a la pregunta sobre cómo golpeaba a los hijos; proporciones más bajas en la justificación de las razones del castigo; selección de menor número de razones $y$, probablemente, tendencia al uso de castigos más

\section{Tabia 5}

Castigo aplicados por colegio. Distribución porcentual




suaves. Estos hechos se suman a la menor proporción de mujeres que reconocieron haber siofo golpeadas por el cónyuge y sugiercn un patrón distunto de violencia familiar, en ese grupo. Dicho patrón no es atribuible a la mayor proporción de hombres en los encuestados de esc colegio; dado que las mujeres de ese grupo también contestaron menos la pregunta incriminatoria. Por otra parte, el sexo de los encuestados insinua mayor participación masculina en las reuniones de padres. Dentro de las etiopatogenjas planteadas para la génesis del maltrato está la que ubica el origen principalmente en el ambiente (génesis ecológica): en esta explicación el maltralo puede desencadenarse $o$ asociarse con apremios familiares, crónicos o agudos (desempleo y situaciones conflictivas familiares), así como tendencia familiar a resolver los conflictos en forma violenta ${ }^{3}$. Los datos del estudio sugieren fuertemente que el castigo físico se asocia con un nivel socioeconómico más bajo y que esta forma de maltrato no seguiría el esquema propuesto por la literatura anglosajona, la que no es categórica en asociar el maltrato con un determinado nivel sociocconómico.

\section{Resumen}

Para conocer opiniones y conductas sobre castigo físico a los niños se aplić́ una encuesta anónima, de respuestas cerradas, a 527 padres y apoderados de Santiago metropolitano: 173 $(32,8 \%)$ de un colegio municipal, $250(45,5 \%)$ de uno subvencionado (ambos graluitos y laicos) y $104(19.7 \%)$ de uno particular, pagado y católico. En este último los padres declararon mejor nivel educacional y hubo una mayor proporción de hombres entre los encuestados. La proporción que reconoció golpcar los niños varió de $56,7 \%$ (particular) a $78,0 \%$ (municipal) y $82 \%$ (subvencionado); a pesar de que proporciones entre $34,1 \%$ (municipal) y $51,9 \%$ (particular) decian que nunca se debía golpearlos. En dos colegios las mujeres reconocieron -con más frecuencia que los hombres- que golpeaban a los nifios ( $\mathrm{p}<0,01$ ). No estudiar, desafiar y cscapar de casa fueron las razones más frecuentes para justificar el castigo física, en los colegios gratuitos; mientras que desafiar lo fue en el particular. Las mujeres tendieron a justificar todas las razones de castigo en porcentajes mayores que los hombres. No hubo diferencias marcadas en los castigos físicos usados, aunque en el colegio particular no se declaró usar correa para golpear. Se concluye que el castigo físico a los niños puede estar asociado con el nivel socioeconómico.

(Palabras clave: maltrato, abuso, violencia, salud familiar.)

\section{Referencias}

1. Vargas $N$, López D, furez $P$, Toro $G$, Zúniga $P$, Ciocca $P$ : Opiniones sobre castigo y castigo físico a niños, de adultos de la región metropolitana. Rev Med Chile 1993; 121: 567-573.

2. Cantrell PJ, Carrica MF, Franklin JN, Grubb $\mathrm{HJ}$. Violent tactics in family conflict relative to familial and economic factors. Psychol Rep 1990; 66: 823. 828.

3. Kim K. Ko B: An incidence survey of battered children in elementary schools of Seoul. Child Abuse Negl 1990; 14: 273-276.

4. Chu JA, DUl DL: Dissociative symptoms in relation to childhood physical and sexual abuse. Am J Psychiatry 1990; 147: 887-892.

5. Poyne MA: Uso y abuso del castigo corporal: una visión caribeña. Child Abuse Negl 1989; 13: 389-401. 